\title{
Optical Calibration Phase Locked Loop for the Shuttle Radar Topography Mission
}

\author{
Dalia A. McWatters, George Lutes, Edward R. Caro, and Meirong Tu
}

\begin{abstract}
The Shuttle Radar Topography Mission (SRTM) is an interferometric synthetic aperture radar system that flew on the space shuttle in February 2000. SRTM has an inboard antenna in the shuttle cargo bay and an outboard antenna at the end of a 60-m mast, extending from the cargo bay. In order to meet the elevation mapping accuracy requirement, the relative phase delay between the radar signals received via the outboard channel, compared with the inboard channel has to be known to within $8^{\circ}$ at 5.3 GHz. This paper describes the design solutions and constraints, the devices, the analysis, and validation used to implement an optical calibration loop for SRTM. The calibration method involves injecting a tone into one panel of the inboard antenna, and sending an optical copy of the tone via a fiber-optic cable to be injected into the outboard antenna. A portion of the optical signal is reflected off an outboard partial mirror and travels back via the fiber to the inboard calibration system. There, it is converted back into a radio frequency tone and its phase is compared with the phase of the original tone. As the temperature of the mast fiber changes, a phase error is detected in the phase comparator. This error is used to control a custom designed optical phase shifter connected in series with the mast fiber. This phase-locked-loop guarantees that the phase of the calibration tone at the outboard stays within $1^{\circ}$ relative to the phase of the calibration tone at the inboard antenna.
\end{abstract}

Index Terms-Interferometric phase calibration, optical phase locked loop, optical phase shifter.

\section{INTRODUCTION}

$\mathbf{T}$ HE Shuttle Radar Topography Mission (SRTM) is a dual polarization, C-band, interferometric synthetic aperture radar. SRTM's mission objective is to create a topographical map of all the Earth's landmass that is accessible to the radar from a $57^{\circ}$ orbit inclination. The radar electronics are mounted to a temperature controlled plate attached to the pallet on the floor of the space shuttle (Endeavor) cargo bay. The inboard antenna array is mounted above the electronics boxes in the cargo bay, and the outboard antenna array is mounted at the end of a 60-m mast extending from the cargo bay, as shown in Fig. 1(a). The antennas are planar active phased arrays with horizontal and vertical polarization sharing the same aperture. This high-efficiency approach requires the transmit/receive electronics to feed directly to the patch elements of each panel [1]. Each C-band panel (sub-aperture) consists of 18 sub-arrays

Manuscript received January 28, 2000; revised October 30, 2000. This work was supported by NIMA (National Imagery and Mapping Agency) contracted to the Jet Propulsion Laboratory (JPL), California Institute of Technology. Reference herein to any specific commercial product, process, or service by trade name, trademark, manufacturer, or otherwise, does not constitute or imply its endorsement by the U.S. Government or JPL, California Institute of Technology.

The authors are with the Jet Propulsion Laboratory (JPL), California Institute of Technology, Pasadena, CA 91109 USA.

Publisher Item Identifier S 0018-9456(01)01186-X. of 18 radiating elements. The inboard antenna consists of 18 panels and performs transmit and receive functions, while the outboard antenna consists of 12 panels and performs receive functions only. In order to meet the elevation accuracy requirement ( $6 \mathrm{~m}$ best case), a challenging phase calibration requirement was imposed on the radar: The relative phase of the signals arriving at the outboard channels has to be known to within $8^{\circ}$ of the phase of the signals arriving at the inboard channels at $5.3 \mathrm{GHz}$. Without compensation or calibration, the phase uncertainty would exceed the requirement by orders of magnitude, because the signals traveling from the outboard antenna pass through RF and microwave devices and coaxial cables whose phase varies with the widely changing temperature environment as the shuttle orbits the Earth. The chosen calibration architecture made use of the high isolation and low loss properties of fiber optics. State-of-the-art opto-electronics components were used and a custom design phase shifter was developed in order to provide the required phase continuous range. The calibration loop functional block diagram is shown in Fig. 1(b).

A calibration tone at $5.3 \mathrm{GHz}$ is produced in the Calibration and Antenna Interface Assembly, which is mounted to the RF Electronics Subsystem (RFES). This tone is coherent with the radar transmit chirp. The calibration tone is routed to the inboard antenna feed network and is injected at the input of one of the panels for $H$ and a different panel for $V$. A copy of the calibration tone is input to the Calibration Optical Transmitter (COT) assembly that is also mounted to the RFES. The COT contains the phase locked loop electronics, a laser transmitter and a receiver, and a custom fiber-optic phase shifter. The loop parameters are designed to track out orbital temperature variations to less than $1^{\circ}$ of phase error. The calibration tone is routed via fiber-optic cable along the mast to the Calibration Optical Receiver (COR). The COR has a partial mirror which reflects a portion of the optical signal back though the mast fiber to the COT where it travels through the phase shifter and circulator into a photo diode to be converted back to RF. This return signal is phase compared with a portion of the original tone and the phase error signal is used to control the phase shifter. The COT can be commanded to turn the laser off, thus disabling the loop. The COT sends loop tracking telemetry to the RFES.

The COR receives the optical calibration tone and converts it back to RF for injection into two of the low noise amplifiers in a single outboard antenna panel for $H$ and $V$. The tone amplitude can be adjusted by command to the COR. The COR reports telemetry about signal presence and temperature.

The equations describing the phase errors as tracked along the loop are discussed in [3]. 


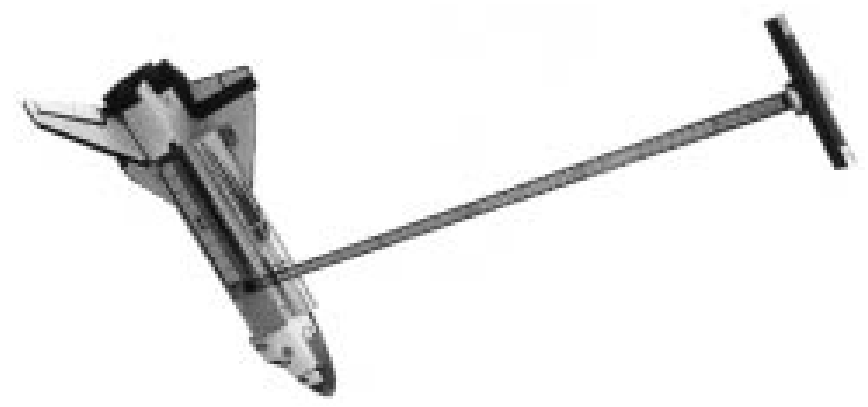

(a)

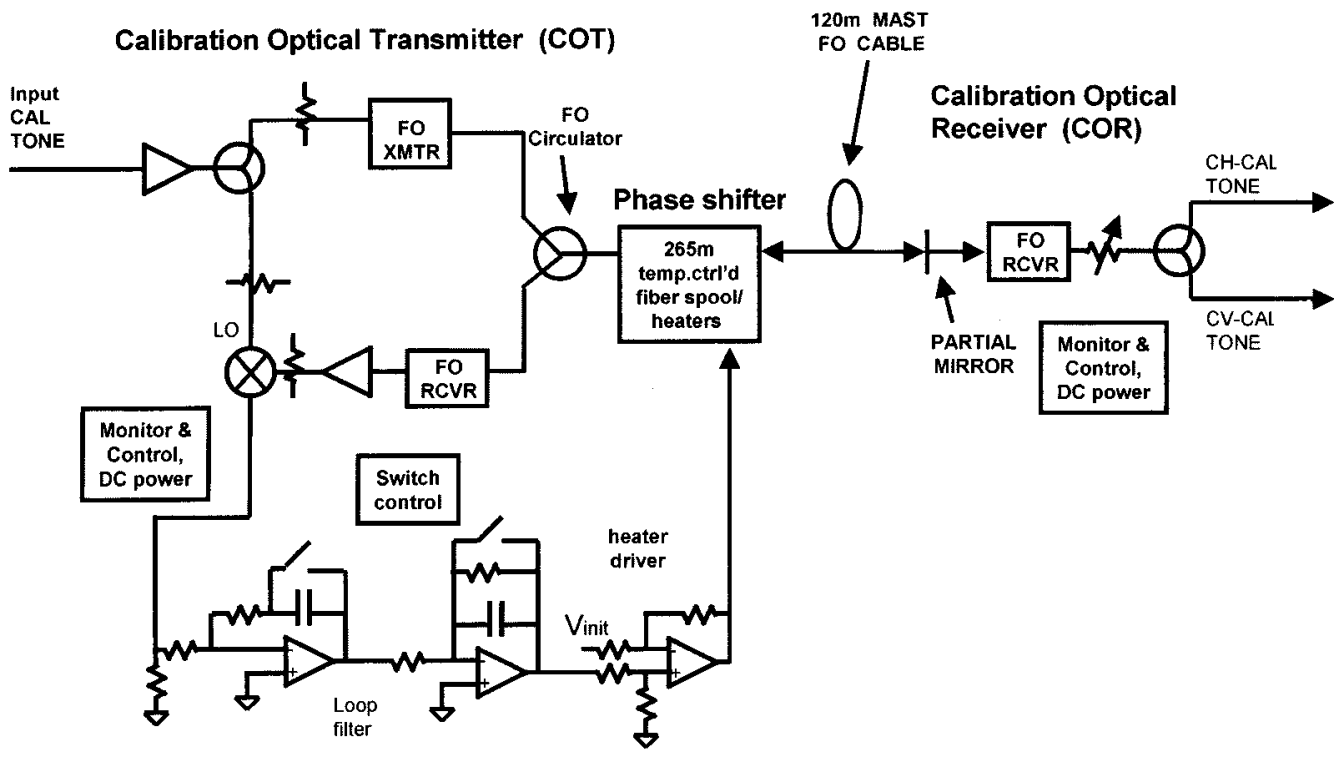

(b)

Fig. 1. (a) SRTM model photo. (b) SRTM optical calibration loop functional block diagram.

\section{CAlibration Loop Design}

The COT and COR utilize high-reliability commercial components for the microwave amplifiers, isolators, mixer, and attenuators. The fiber optics transmitter module is a Uniphase Telecommunications Products (UTP) Small Integrated Transmitter Unit (SITU), which consists of a 1550-nm high-power single-mode CW laser followed by a Mach-Zehnder modulator [3]. The 3-port circulator is a commercial device fabricated by E-Tek Dynamics. It has an insertion loss of $<1.3 \mathrm{~dB}$, minimum isolation of $45 \mathrm{~dB}_{\mathrm{o}}$ (i.e., $90 \mathrm{~dB}$ when detected and converted to $\mathrm{RF}$ ) and optical return loss of better than $50 \mathrm{~dB}_{\mathrm{o}}$. The partial mirror in the COR is a Faraday rotating half mirror from E-Tek. The 120-m mast fiber-optic cable is a single-mode fiber from Sumitomo Electric Industries. The fiber was fabricated using a novel liquid crystal polyester and nylon secondary coating materials jacketed over a soft silicone primary coated fiber. This secondary coating resin has the opposite coefficient of thermal expansion compared to glass, so the result is a fiber with extremely low coefficient of delay. However, this fiber-optic cable is extremely brittle, and susceptible to damage due to sharp bends/kinks. The fiber was jacketed with a Teflon braid and inserted into a corrugated plastic tube with a lengthwise slit. The tube was tied at each end to the AVIM (manufactured by Diamond) connector body.
The following describes the operation of the optical calibration loop. When the loop is powered on, the loop filter switches are commanded to be closed, thus discharging the capacitors and setting the heater drivers to the middle of their range. The laser is given time to stabilize. To enable the loop, the loop filter switches are opened and the loop acquires and achieves lock.

The control loop was designed to compensate for a temperature cycle that equals the shuttle orbit period of $90 \mathrm{~min}$. SRTM thermal analysis predicted that the mast fiber would experience $12^{\circ} \mathrm{C}$ peak to peak sinusoidal variation of temperature over $45 \mathrm{~min}$. Taking into account the mast fiber length and thermal coefficient of delay, this would require the phase locked loop to compensate for $7.2^{\circ}$ of phase over $45 \mathrm{~min}$. During acquisition, the loop would need to be able to lock with an initial phase offset of up to $360^{\circ}$.

The key design parameters were the performance of the phase shifter and the loop filter.

\section{A. Phase Shifter Design}

The phase shifter functional diagram is shown in Fig. 2(a). A photo of the spool in the test set up is shown in Fig. 2(b). The spool is made of an aluminum coil form, with six layers of fiber wound and coated with Norland 81 UVacrylate. Kapton 


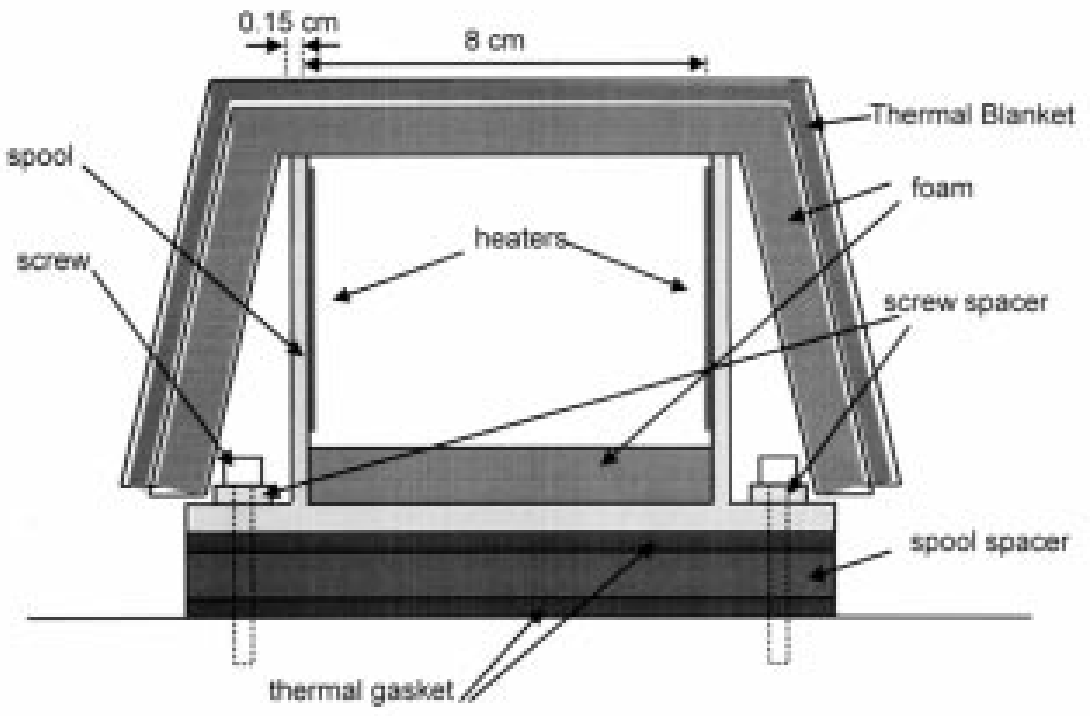

(a)

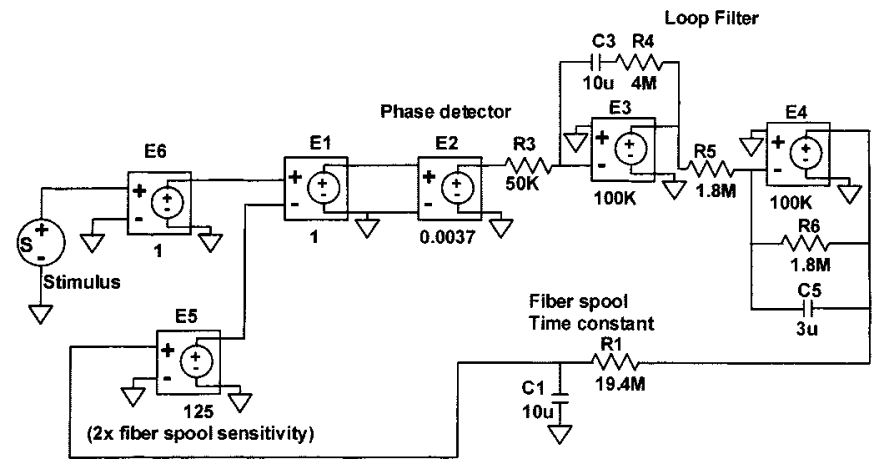

(c)

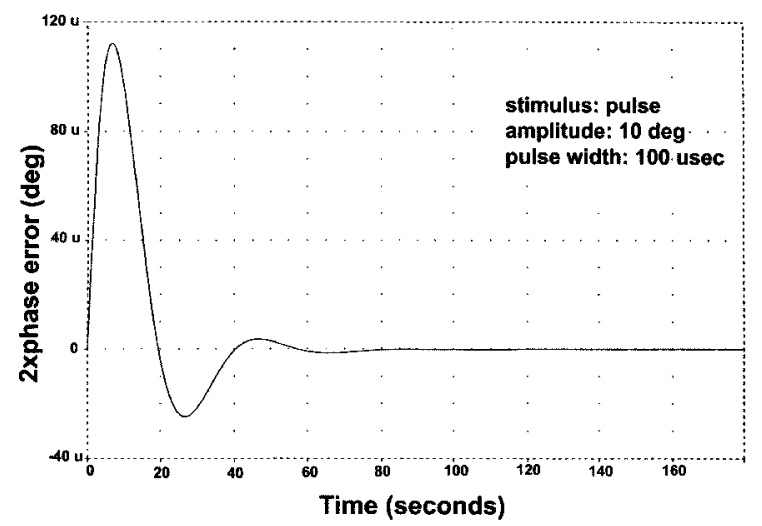

(e)

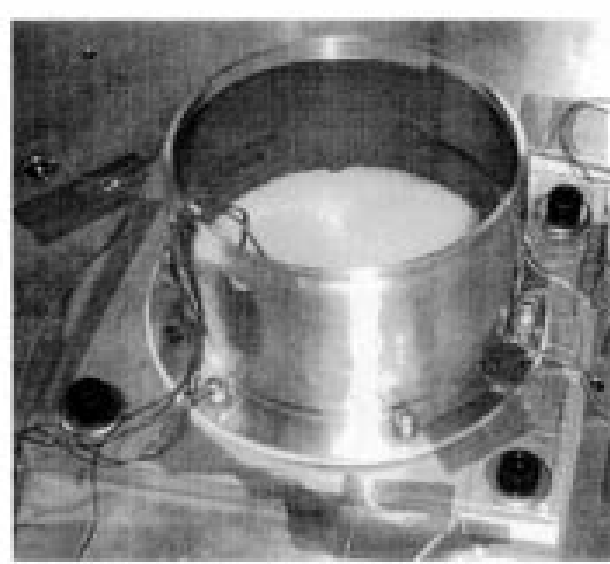

(b)

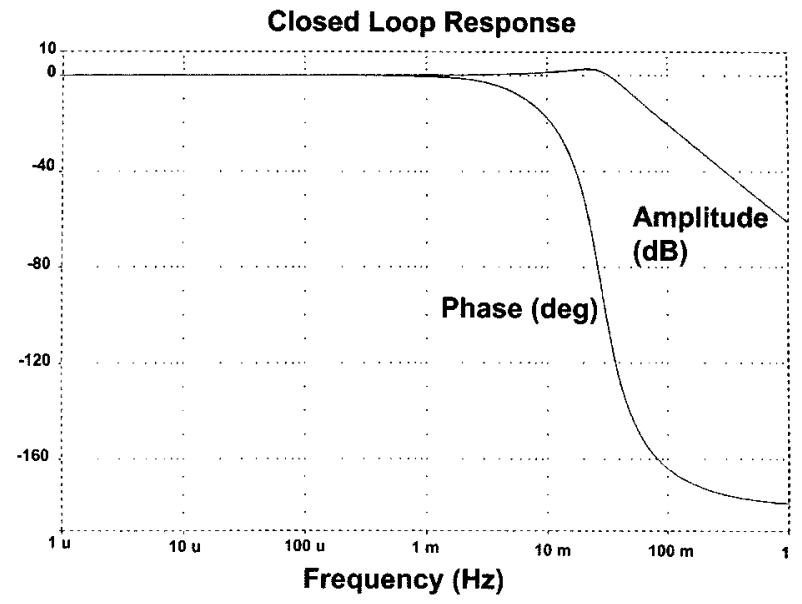

(d)

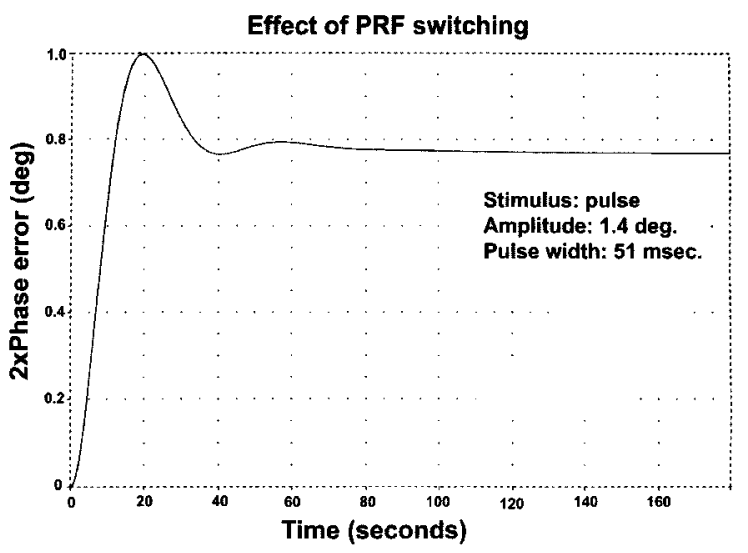

(f)

Fig. 2. (a) SRTM optical calibration loop fiber phase shifter diagram. (b) SRTM optical calibration loop fiber phase shifter photo. (c) SRTM optical calibration loop spice model. (d) SRTM optical calibration loop spice simulation of closed loop bandwidth. (e) SRTM optical calibration loop spice simulation of pulse response. (f) SRTM optical calibration loop spice simulation of changing pulse repetition rates.

Thermofoil heaters (Minco) with pressure sensitive adhesive are attached to the inner surface of the spool. A fiberglass spacer is placed between the spool flange and the mounting plate, providing a known thermal resistance. In order to improve the thermal interface between the fiberglass and the aluminum, thermal gaskets are inserted above and below the 
fiberglass spacer. In order to prevent stray thermal paths, each mounting screw is isolated by a fiberglass washer and torqued to a specified value. A thermal blanket covers the fiber spool in order to prevent thermal radiation between the fiber spool and the COT housing. Polyimid foam is installed between the coil form and the thermal blanket in order to reduce thermal conduction between the fiber spool and the thermal blanket and also to minimize heat conduction during testing in air.

The fiber-optic spool design parameters were optimized according to the following relationships and constraints.

a) Phase shifting range is directly proportional to the length of the spool fiber.

b) Phase shifter sensitivity, i.e., the rate of phase change with respect to temperature is directly proportional to the length of the spool fiber.

c) Coil form dimensions and material have to fit within the mass, size and rigidity packaging constraints imposed by the mechanical design of the system.

d) Fiber spool thermal mass is directly proportional to the thermal time constant. This limits the size and thickness of the coil form and the length of the fiber.

e) Spool spacer thickness is directly proportional to the thermal time constant. The thinner the spacer, the shorter the time constant (i.e., faster response which is desirable).

f) The temperature difference (delta- $T$ ) between the spool flange and the cold plate is directly proportional to the spool heater power, and inversely proportional to the spool spacer thickness. i.e., more heater power would be required for a thin spacer (but electrical power is at a premium on a space-borne instrument) in order to achieve the required delta- $T$. The higher the delta- $T$, the faster the cooling/heating rate of the spool.

The fiber spool assembly was mounted to a temperature-controlled plate and characterized in a vacuum chamber. The heater driver was set to full level, bringing the temperature to the maximum delta- $T$ achievable by the driver/spool configuration. Then, the driver was turned off. During the heating and cooling, the phase of the signal going through the fiber was measured using a network analyzer, and the temperature on various places on the spool was measured. The time constant was thus measured as well as the max. delta- $T$.

The final time constant (in vacuum) was measured at $194 \mathrm{~s}$ with a maximum delta- $T$ of $18^{\circ} \mathrm{C}$. The temperature range required for acquisition (of $\pm 360^{\circ}$ phase) was $6^{\circ} \mathrm{C}$ and the minimum delta- $T$ required for heat transfer rate was $5{ }^{\circ} \mathrm{C}$ in the heating direction and $5^{\circ} \mathrm{C}$ in the cooling direction. This leaves $2{ }^{\circ} \mathrm{C}$ of margin.

Once the spool/spacer performance achieved the required range, rate, and margins, it was time to begin designing the loop filter.

\section{B. Control Loop Design and Simulation}

The loop filter poles and zeros and dc gain were selected in order to achieve the desired slope at the crossover frequency of the open loop response (i.e., to obtain sufficient phase margin for stability of the loop).
The lowest frequency pole of the loop is the fiber spool (with its fiberglass spacer) at about $1 \mathrm{MHz}$, with a time constant of approximately a fifth of the flight temperature cycle period (90 min orbit).

A zero was added at $4 \mathrm{MHz}$, and another pole was added at $29 \mathrm{MHz}$. These were implemented with active filters using operational amplifiers.

The loop was modeled in Spice [Figs. 2(c) and (d)] and simulations were run to characterize loop performance, with the following results.

a) Exponential cooling of the mast after deployment from the shuttle cargo bay during hours 2-13, is tracked by the loop to within 28 mdeg. (Note: the actual mapping begins after the temperature has stabilized, but some on-orbit tests are performed during the cooling period.)

b) Orbital temperature cycle is tracked by the loop to within 50 mdeg.

c) The calibration tone generated by the RFES contains periodic phase resets and pulsed chirps at alternating pulse repetition rates. The phase resets are too short to affect the loop. Fig. 2(e) plots the round trip phase error calculated by the Spice model. At the beginning of a "mapping data take," the pulse repetition rate will begin to alternate. This may cause the loop to adjust its average phase by a fraction of a degree. [Worst case is shown in Fig. 2(f).]

d) The performance of the optical calibration loop imposed certain requirements on the operation of its "parent": the RFES. The calibration tone is to be powered on at all times, even between data takes, in order to avoid tens of seconds required by the loop to stabilize.

e) The calibration tone frequency is to be held constant and not allowed to sweep its frequency at the beginning of each data take, again in order to avoid up to $60 \mathrm{~s}$ of loop settling time.

Simulations showed that the design would meet requirements. It was now time to begin testing of the hardware.

\section{TESTING AND VERIFICATION}

\section{A. Thermal Vacuum}

The COR, COT, and a flight-like mast fiber, were tested as a system, inside a thermal-vacuum chamber according to the configuration shown in Fig. 3(a). The COT was mounted to a temperature-controlled plate similar to the way it is mounted to the RFES. The COT and its plate were thermally blanketed to prevent radiating to the other elements in the chamber. The COR, which is painted black, is designed to radiate heat to the outboard structure environment. In the thermal-vacuum chamber, the COR was suspended inside a shroud (a black temperature controlled cylinder). The mast fiber was rolled as a loose bundle and placed inside a black box, which was mounted to another temperature-controlled plate. The fiber box and its plate were thermally blanketed as well.

Temperature sensors were placed in the fiber bundle and its box, as well as on the outer surfaces of the COR, COT, and 


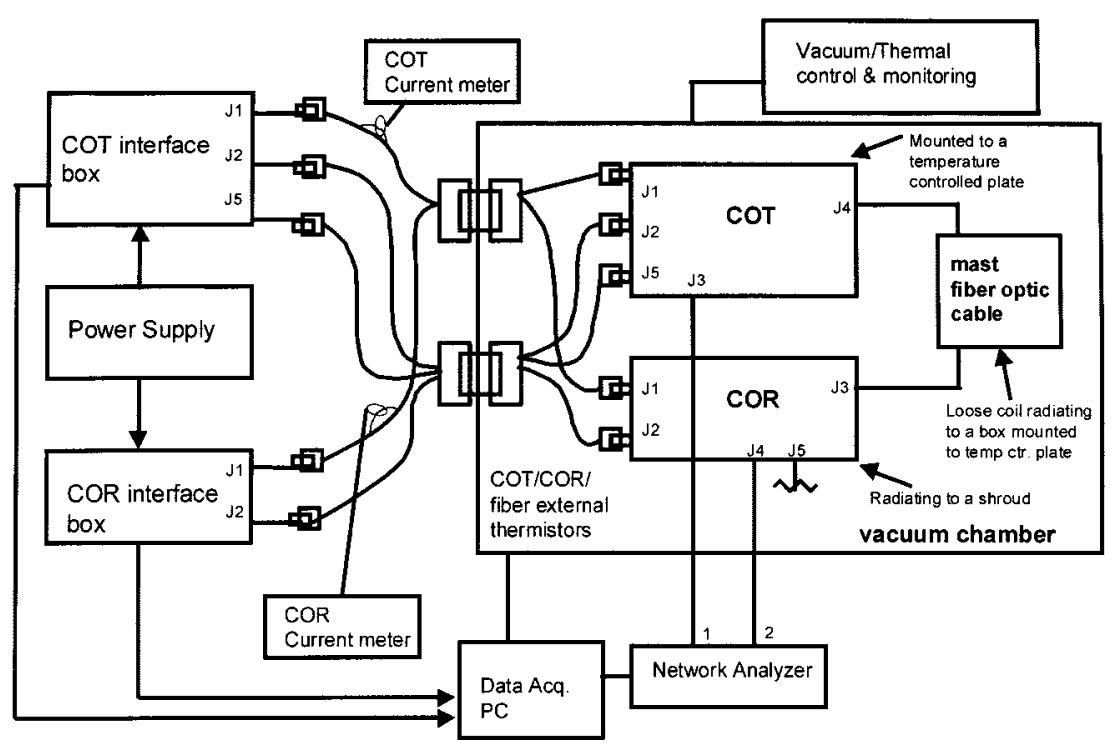

(a)

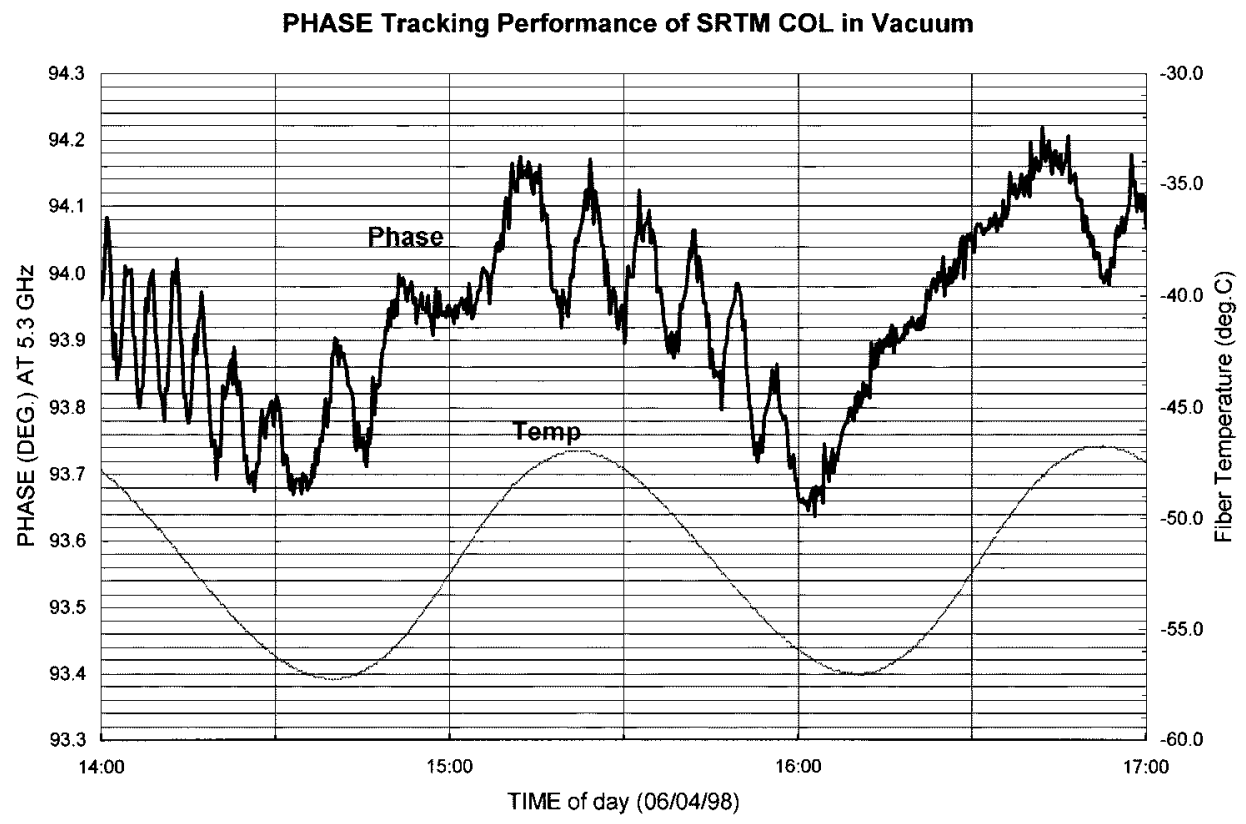

(b)

Fig. 3. (a) SRTM optical calibration loop thermal vacuum test configuration. (b) SRTM optical calibration loop performance in vacuum.

the input/output coax cables. (The coax cables were tested separately and their phase versus temperature characteristics were taken into account during the interpretation of the thermal vacuum test results.)

The COL performance was monitored via the telemetry signals reported by the COT: the loop phase error and the heater drive voltage as well as internal temperature measurements such as delta- $T$ and laser temperature.

The calibration tone source was supplied by the network analyzer which measured the difference in phase between the input to the COT and the output of the COR. The telemetry and the network analyzer phase and amplitude data were collected by a computer for archive and display. The first stage of the test ver- ified functionality in vacuum, with the COR, COT and the mast fiber each at its nominal flight temperature.

Next, the mast fiber temperature was cycled according to predicted orbital variation, at the coldest expected range (for worst case phase performance). The loop phase error, as shown in Fig. 3(b), is less than $0.6^{\circ}$. Finally, the temperatures of the COR and the COT were each varied over its expected mission range, one at a time while the mast fiber temperature was cycling. This verified that the loop would track over the entire mission.

\section{B. Radiation Environment}

The total ionizing dose radiation expected for the 11-day mission of SRTM is a few hundred rads for 1 mil of aluminum 
shielding. The coating of the mast fiber was analyzed and measured [2] and the conclusion was that the coating would provide shielding equivalent to 8 mils of aluminum, where 1 mil of aluminum was deemed sufficient. The rest of the electronics, under 100 mils of aluminum, is extremely well protected from the radiation environment.

\section{Vibration}

The COR and COT passed vibration tests, simulating the vibration levels during the space shuttle launch. The vibration test included random vibrations of specified levels over a specified spectrum with an overall $g_{\mathrm{rms}}=15.6$ in plane and 11.0 out of plane for the COR, 12.2 in plane and 22.0 out of plane for the COT.

\section{D. $E M C$}

The COR and COT passed Electro-Magnetic-Compatibility (EMC) susceptibility tests under simulated levels of conducted and radiated emissions from the shuttle. The radiated and conducted emissions of the optical calibration loop subsystem were also characterized, verifying that they do not exceed the levels tolerated by the shuttle electronic systems.

\section{SRTM PHASE CALIBRATION}

The calibration tones injected into the outboard horizontal polarization channel and vertical polarization channel and the inboard horizontal and vertical polarization channels become imbedded in these four radar channels carrying the received echoes from the ground. The radar system uses additional methods in order to occasionally determine the absolute phase difference between the radar channels. However, in order to improve the height resolution, knowledge of the relative phase changes over temperature during a mapping pass, is made available by the Calibration Optical Loop. Post processing of the radar echo data essentially subtracts the phase variations of the calibration tone from the echo in each channel, thus reducing the effect of phase changes that are internal to the radar system. This allows for a more accurate reconstruction of terrain height ( $6 \mathrm{~m}$ best case).

The optical calibration loop phase error was required not to exceed $3.4^{\circ}(1.6$ sigma) during a 40 min mapping data take during the mission. The corresponding expected performance, based on the thermal vacuum measurements, is $2.4^{\circ}$ (taking into account the expected temperature variations in the COT, COR, and the mast fiber). However, the optical calibration loop phase performance is only one component of the overall phase error of the SRTM calibration.

In order to calculate the total interferometric phase uncertainty, other contributing factors are taken into account. For example, the coax cables injecting the calibration tone into the inboard antenna panels have been characterized over temperature in the lab. During the mission their temperatures will be logged, and their phase variation will be later extracted from the received calibration tone phase. The inboard antenna panels phase versus temperature characteristics were also measured, and their predicted phase versus temperature variations during the mission will be extracted since the inboard antenna is not included in the calibration tone injection path. In the outboard antenna, the calibration tone is injected into only one of the panels, so the difference in phase versus temperature performance between the 12 outboard antenna panels, was also characterized.

\section{A Highly SuCCESSFUl Mission}

The SRTM launched on February 11, 2000 aboard the space shuttle Endeavour. During its 11-day mission, it successfully met its objective of mapping $80 \%$ of the Earth landmass (between $60^{\circ}$ North and $56^{\circ}$ South) with 30-m postings, with better than 16-m absolute vertical height accuracy and with better than 10-m relative accuracy (accuracies quoted hold for $90 \%$ of the terrain). The radar systems worked flawlessly, including the Calibration Optical Loop that stayed locked for the entire duration of the mission.

\section{SUMMARY AND CONCLUSIONS}

An optical calibration phase-locked loop was successfully designed, simulated, implemented, and tested for the SRTM, providing a delivery system for a $5.3 \mathrm{GHz}$ tone of a known relative phase to be injected into the outboard antenna system at the end of a 60-m mast. The design made use of state-of-the-art opto-electronics components as well as a custom-designed optical phase shifter. The phase of the calibration tones embedded in the radar receive channels will be used to enhance the accuracy of the ground echo phase information, which is used to calculate the elevation of the mapped terrain. The SRTM completed its mapping objectives with great success and is now engaged in an 18-month effort to process the data and generate the most complete, consistent, and accurate topographic map of the Earth land mass.

\section{ACKNOWLEDGMENT}

The authors' most sincere thanks and appreciation go to Dr. B. Lurie (JPL) for the control loop design guidance, L. Veilleux (JPL) for technical support of design compatibility between the COL and the C-Radar instrument, B. Finamore (JPL) for the Data Acquisition and test support, L. Maleki (JPL) for support, R. Logan and his team (Uniphase) for dedication in implementation and fabrication of the flight calibration loop subassemblies, and to the entire SRTM team for support in testing and integration.

\section{REFERENCES}

[1] R. L. Jordan, "The SIR-C/X-SAR synthetic aperture radar system," Proc. IEEE, vol. 79, pp. 827-838, June 1991

[2] G. Lutes, "Radiation darkening of the SRTM optical fiber,", JPL Interoffice Memo. 335.10-97-013, Oct. 23, 1997.

[3] G. Lutes, D. McWatters, and M. Tu, "A 60 meter delay stabilized microwave fiber optic link for $5.3 \mathrm{GHz}$ reference signal distribution on the shuttle radar topography mapper," in PSAA 10th DARPA Symp., Feb. 1999. 


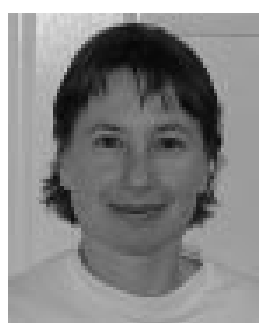

Dalia A. McWatters received the B.Sc. degree in electrical engineering from California State University, Long Beach, in 1985 and the M.Sc degree in electrical engineering from University of California, Los Angeles, in 1989.

Since 1985, she has been with the Jet Propulsion Laboratory (JPL), Pasadena, working on various projects involving design, analysis, and testing of ground and flight Radio Frequency (RF) subsystems. She was the RF Electronics Subsystem Lead Engineer for the Shuttle Radar Topography Mission for which her responsibilities included design, integration, and testing of the dual-frequency and dual-polarization receiver and transmitter, beam auto-tracker, and the calibration optical phase locked loop. Her responsibilities progressed into testing and integration with the space shuttle at Kennedy Space Center and finally, into mission operation in Houston, TX, during the successful 11-day flight.

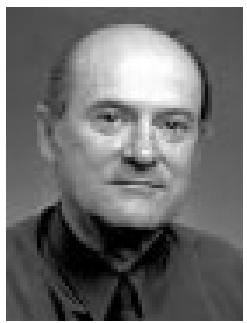

George Lutes studied electrical engineering at various colleges and universities since 1958 while working in the aerospace industry.

From 1958 to 1960 , he was with Autonetics, where he worked on radar systems for the F-104 fighter aircraft. From 1961 to 1963, he worked on a remote sensing space program at TRW, where he helped develop transistor power amplifiers. Since 1964, he has been with the Jet Propulsion Laboratory (JPL), Pasadena. From 1964 to 1978, he designed circuitry for ultrahigh stability frequency and timing systems for the NASA/JPL Deep Space Network (DSN). In the late 1970's and early 1980's, he pioneered the field of analog fiber-optic systems. He installed the world's first operational single-mode fiber-optic system. This system was used to distribute ultrastable frequency references between Deep Space Stations in the Deep Space Complex located in the Mojave Desert. It is still in operation. In recent years, he has developed systems to reduce delay variations in fiber-optic links. This work led to the fiber-optic system used on SRTM that is described in this paper.

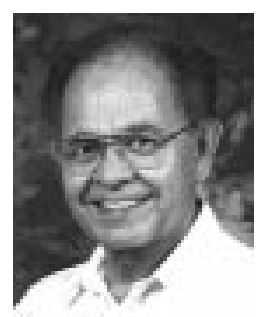

Edward R. Caro received the diploma in electrical engineering from the National Institute of Technology, The Philippines, and attended the Graduate School of Management, University of California, Los Angeles.

For the last few decades, while working at the Jet Propulsion Laboratory, Pasadena, CA, he has been a leader in developing the technology for airborne and spaceborne SAR systems for 2-D and 3-D mapping. His work resulted in several successful space shuttle radar-mapping missions.

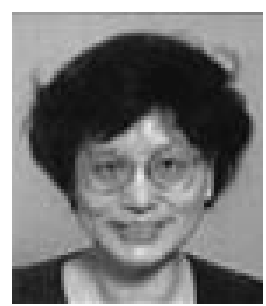

Meirong Tu received the M.S. and Ph.D. degrees in engineering mechanics from the Chinese Academy of Sciences, Beijing, in 1983 and 1986, respectively.

She was a Post-Doctoral Research Fellow at the Virginia Polytechnic Institute, Blacksburg, from 1987 to 1988 . While pursuing the doctoral degree, she was engaged in various interferometry techniques for micro-deformation measurement and stress and strain analysis. She was with the Florida State University, Tallahassee, as a Research Scientist, and joined the Jet Propulsion Lab, Pasadena, in 1997 as a Photonics Specialist. She is currently with General Photonics Company as a Senior Engineer developing optical fiber components and devices. 